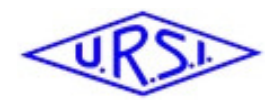

\title{
An Inverse Scattering Procedure in Lebesgue Spaces with Non-Constant Exponents
}

\author{
Claudio Estatico ${ }^{(1)}$, Alessandro Fedeli ${ }^{(2)}$, Matteo Pastorino ${ }^{(2)}$, and Andrea Randazzo ${ }^{(2)}$ \\ (1) Department of Mathematics, University of Genoa, Genoa, Italy \\ (2) Department of Electrical, Electronic, Telecommunications Engineering, and Naval Architecture \\ University of Genoa, Genoa, Italy
}

\begin{abstract}
Within the ever-growing field of electromagnetic imaging, inversion procedures are conventionally described in the mathematical framework of Hilbert spaces. Usually, the over-smoothing effects and oscillations that arise using a Hilbert-space formulation make the dielectric reconstruction of targets inaccurate. This problem is strongly reduced by the recent development of inversion techniques in Banach spaces. However, the selection of the Banach space norm parameter is critical for obtaining precise reconstructions, and no exact rules exist for this choice. To overcome this issue, an innovative approach in variable exponent Lebesgue spaces is proposed here, along with a preliminary numerical validation.
\end{abstract}

\section{Introduction}

The development of electromagnetic inverse scattering methods is an active research field in the scientific community. In particular, the discovery of several novel applications of inverse scattering, ranging from biomedical and industrial systems [1]-[3] to geophysical inspection devices [4], [5], motivate the need for efficient highperformance methods. In many situations, the accuracy of the inverse scattering procedure in reconstructing the dielectric properties of the target under test is crucial. However, the electromagnetic inverse problem, without the introduction of simplifying approximations, is still considered very hard to solve [6]. A particularly efficient family of deterministic inversion techniques is the GaussNewton one, in which the initially nonlinear problem is firstly linearized and then solved in a regularized way [7][9]. These solution algorithms are usually described in the mathematical framework of Hilbert functional spaces. Despite the simplified treatment of such spaces, the obtained reconstructions are often unable to correctly detect sharp discontinuities in the dielectric properties of targets, and both ringing effects and artifacts usually corrupt the retrieved images, especially in the background. In order to overcome these problems, an inverse scattering method working in the more general framework of Banach spaces has been recently formulated [10] and tested in different scenarios [11], [12]. However, an unresolved issue still exists: the choice of the parameter $p$ that defines the working $L^{p}$ Banach space and its norm. Indeed, by several numerical evidences in $L^{p}$ spaces, restoration in spaces with small exponents $p>1$ are very useful to reduce ringing effects in the background and to restore the discontinuities between different dielectric objects, whereas resolution with $p \approx 2$ improves the quality of the restoration inside the scatterers. Aimed to overcome this issue, in the present paper, we propose for the first time an inverse scattering procedure that works in the context of variable exponent $L^{p(\cdot)}$ Lebesgue spaces. In this case, instead of being fixed, the $p$ parameter can be different for different parts of the investigation domain, i.e., the region that is supposed to contain the target under test.

This contribution has the following structure. In Section 2, an overview of the formulation of the novel method is presented. In addition, Section 3 reports some preliminary numerical simulations concerning the reconstruction of cylindrical objects in free space. Conclusions follow.

\section{Formulation of the Method}

In our inverse scattering problem, an infinite cylindrical geometry under transverse-magnetic (TM) illumination is considered. Hence, we are interested in restoring the twodimensional (2D) configuration of an unknown scattering object, enclosed in a 2D investigation bounded domain $\Sigma \subset$ $\mathbb{R}^{2}$. In an observation domain $\Omega \subset \mathbb{R}^{2}$, a transmitting antenna illuminates the investigation domain with a known time-harmonic TM-z polarized electromagnetic field with angular frequency $\omega$, and simultaneously a set of receiving antennas measures the corresponding scattered field. A multiview approach, where the position of both the transmitting and the receiving antennas is subsequently changed, is considered.

Our goal is to find, by exploiting the measured scattered field data, the so-called contrast function inside the investigation domain $\Sigma$, that is,

$$
x(\mathbf{r})=\epsilon_{r}(\mathbf{r})-\frac{j \sigma(\mathbf{r})}{\omega \epsilon_{0}}-1, \quad \mathbf{r} \in \Sigma
$$

where $\epsilon_{r}(\mathbf{r})$ and $\sigma(\mathbf{r})$ are the relative dielectric permittivity and the electric conductivity, respectively, and $\epsilon_{0}$ is the vacuum permittivity. In the discretized version of the problem, the unknown is represented by a vector $\mathbf{x}=$ $\left[x\left(\mathbf{r}_{1}\right), \ldots, x\left(\mathbf{r}_{N}\right)\right], \mathbf{r}_{i}(i=1, \ldots, N)$ being the locations of the $N$ subdomains in which the investigation region $\Sigma$ is subdivided. By considering the analytical formulation developed in [10], [11], the electromagnetic problem can be written in an operator form as 


$$
\mathbf{F}(\mathbf{x})=\mathbf{y}
$$

in which $\mathbf{F}: X \rightarrow Y$ denotes the nonlinear scattering operator mapping the dielectric properties of the object $\mathbf{x} \in X$ on the generated scattered electric field, and $\mathbf{y} \in Y$ is a vector containing all the measured scattered electric field data in the observation domain.

To solve (2), a regularizing Newton-like iterative method is applied to minimize the residual functional $\Psi: Y \rightarrow \mathbb{R}$ defined as follows

$$
\Psi(\mathbf{x})=\frac{1}{2}\|\mathbf{F}(\mathbf{x})-\mathbf{y}\|_{Y}^{2},
$$

where $\|\cdot\|_{Y}^{2}$ denotes the square of the norm of the functional space $Y$. The basic steps of the minimization procedure are the following.

1. Initialize a null starting guess $\mathbf{x}_{0}=\mathbf{0} \in X$. Set $n=0$.

2. Set $n \leftarrow n+1$. Linearize (2) at the current estimate $\mathbf{x}_{n} \in X$ and consider the linear problem

$$
\mathbf{F}_{n}^{\prime} \mathbf{h}=\mathbf{y}-\mathbf{F}\left(\mathbf{x}_{n}\right)
$$

where $\mathbf{F}_{n}^{\prime}: X \rightarrow Y$ is the Fréchet derivative of $\mathbf{F}$ at $\mathbf{x}_{n}$ [10].

3. Compute a regularized solution $\tilde{\mathbf{h}} \in X$ of (4) by means of a truncated Landweber algorithm [13] associated to the two functional spaces $X$ and $Y$.

4. Update the current solution with $\mathbf{x}_{n+1}=\mathbf{x}_{n}+\tilde{\mathbf{h}}$.

5. Iterate steps $2-4$ until a proper convergence criterion is fulfilled.

We remark that the formulation of the problem in different spaces $X, Y$ yields to different solution methods, since the associated norms are different. In particular, the Landweber method for the solution of the linearized functional equation at step 3 strictly depends on the choice of the functional spaces $X$ and $Y$. In the conventional solving schemes, $X$ and $Y$ are $L^{2}(\Sigma)$ and $L^{2}(\Omega)$ respectively, being $L^{2}$ the Hilbert spaces of squareintegrable functions, leading to the classical well-known least square minimization [10]. Recently, regularization schemes in the $L^{p}$ Lebesgue spaces of $p$-integrable functions, with $1<p<2$, have been introduced in [10]. Indeed, it has been shown that the resolution of functional equations in these spaces reduces over-smoothing effects of the classical $L^{2}$ solutions, leading to a better localization of the discontinuities of the scatterers. We recall that the $L^{p}$-norm of a vector $\mathbf{u}=\left(u_{1}, u_{2}, \cdots, u_{N}\right)$ of length $N$, for $1 \leq p<+\infty$, is defined as follows

$$
\|\mathbf{u}\|_{L^{p}}=\sqrt[p]{\left|u_{1}\right|^{p}+\left|u_{2}\right|^{p}+\cdots+\left|u_{N}\right|^{p}},
$$

In this paper, we consider for the first time a different class of Lebesgue spaces, namely the variable exponent Lebesgue spaces $L^{p(\cdot)}$. Differing from the conventional $L^{p}$ Lebesgue spaces of $p$-integrable functions, in a $L^{p(\cdot)}$ space, the power $p$ used in the $p$-norm is not constant, but it is a function $p(\cdot)$ of the domain (that is, in our discrete context, a vector of different elements). Hence, these spaces allow us to set different values of the parameter $p$ in the domain, in order to better control the sparsity in the background, with values of $p$ close to 1 , and to better estimate the dielectric parameters inside the scattering objects, with values of $p$ close to 2 .

Variable exponent Lebesgue spaces $L^{p(\cdot)}$ have a much more involved structure than classical, i.e., constant exponent, Lebesgue spaces $L^{p}$. Indeed, given a vector of power values $\mathbf{p}=\left(p_{1}, p_{2}, \cdots, p_{N}\right)$, with $1 \leq p_{i}<+\infty$ for any $i$, the associated norm requires first the computation of the following quantity, namely the so-called modulus,

$$
\rho(\mathbf{u})=\left|u_{1}\right|^{p_{1}}+\left|u_{2}\right|^{p_{2}}+\cdots+\left|u_{N}\right|^{p_{N}},
$$

and then the solution of the following one dimensional minimization problem

$$
\|\mathbf{u}\|_{L^{p(\cdot)}}=\inf \left\{\gamma>0: \rho\left(\frac{\mathbf{u}}{\gamma}\right) \leq 1\right\} .
$$

It is interesting to notice that this norm is a natural extension of the conventional norm (5). Indeed, as expected, for a vector of constant values $\mathbf{p}=(p, p, \ldots, p)$, we have that $\|\mathbf{u}\|_{L} p(\cdot)=\|\mathbf{u}\|_{L} p$.

As regards the choice of the vector $\mathbf{p}$ inside the regularization procedure, in the first Gauss-Newton iteration a vector of constant values $\mathbf{p}=(2,2, \ldots, 2)$ has been used, under the hypothesis of no a-priori information about the targets under inspection. Then, the obtained reconstruction result is exploited for building a map of the values $\mathbf{p}$, which linearly connects the normalized absolute value of the retrieved contrast function to a predefined range of $p$ values, with $1<p \leq 2$. This map is then updated at each outer iteration.

\section{Numerical Results}

The reconstruction algorithm described in the previous Section has been validated in a simulated environment. A single operating frequency $f=300 \mathrm{MHz}$ has been considered. The scattered field data have been computed by means of a custom numerical code based on the method of moments [14]. Before the inversion, the simulated data have been corrupted by an additive Gaussian noise with mean value equal to zero and a signal-to-noise ratio $S N R=$ $20 \mathrm{~dB}$. A square inversion domain $\Sigma \subset \mathbb{R}^{2}$ of side length $D=2 \lambda$ ( $\lambda$ being the free space wavelength) is supposed to contain the scatterer's support. A set of $S=50$ linecurrent sources, located on a circumference of radius $\rho=$ $1.5 \mathrm{~m}$ at the points

$$
\mathbf{r}_{s}^{T X}=\left(\rho \cos \theta_{s}^{T X}, \rho \sin \theta_{s}^{T X}\right), \quad s=1, \ldots, S
$$

where $\theta_{s}^{T X}=2 \pi(s-1) / S$, illuminate the investigation region. For the $s$ th view $(s=1, \ldots, S)$ the observation domain $\Omega$ comprises $M=S-1=49$ measurement points positioned at

$$
\mathbf{r}_{m, s}^{R X}=\left(\rho \cos \theta_{m, s}^{R X}, \rho \sin \theta_{m, s}^{R X}\right), \quad m=1, \ldots, M
$$




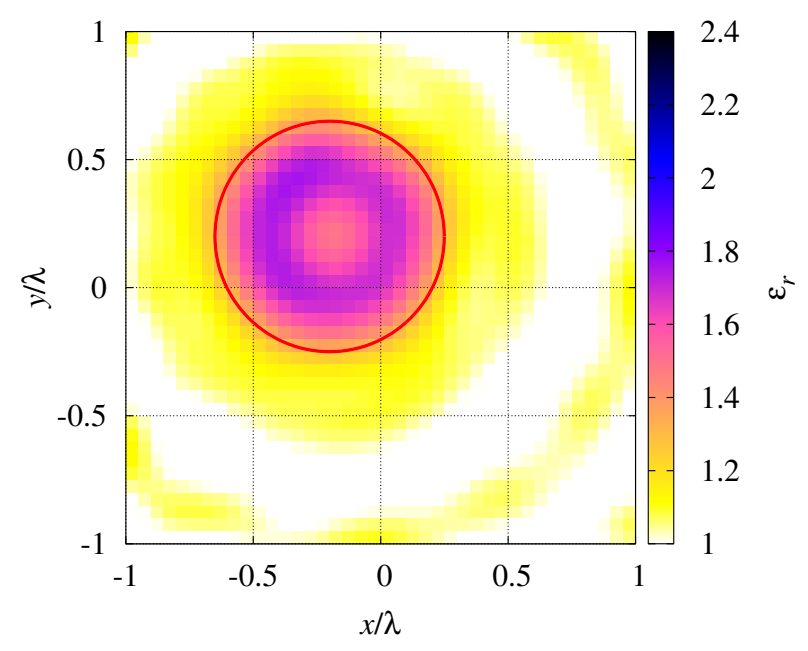

Figure 1. Reconstructed distribution of the relative dielectric permittivity $\epsilon_{r}$ inside the investigation domain. Red circle marks the true profile. Hilbert-space reconstruction.

with $\theta_{m, s}^{R X}=\theta_{s}^{T X}+\theta_{0}+2\left(\pi-\theta_{0}\right)(m-1) /(M-1)$.

For solving the inverse problem numerically, a discretization of the investigation domain into $N_{D_{i n v}}^{i n v}=$ $40 \times 40$ square subdomains has been introduced. It is worth noting that a finer discretization $N_{D_{i n v}}^{f w d}=63 \times 63$ has been used in the solution of the forward problem, for avoiding inverse crimes. The background propagation medium is assumed to be vacuum (i.e., $\epsilon_{r}=1, \sigma=$ $0 \mathrm{~S} / \mathrm{m})$. All the targets in the present analysis are supposed to be infinite cylinders perpendicular to the $x y$ plane. The target is a lossy dielectric cylinder with circular cross section. It is centered at $\mathbf{r}_{c}=(-0.2,0.2) \lambda$, and is characterized by a diameter $d_{c}=0.9 \lambda$, relative dielectric permittivity $\epsilon_{r}=2$, and electric conductivity $\sigma=$ $0.01 \mathrm{~S} / \mathrm{m}$. Simulated inversions by using three different methods belonging to the same Gauss-Newton family have been performed: the proposed algorithm with non-constant exponent has been compared with the standard Hilbertspace reconstruction and a more recent Banach-space inversion procedure. In all methods, $K_{L W}=5$ inner Landweber iterations and $K_{G N}$ Gauss-Newton steps have been executed. In the novel variable exponent method, the range of variation of $p(\cdot)$ has been chosen between 1.4 and 2.0. The reconstruction obtained in the standard framework of Hilbert spaces (i.e., Banach spaces with $p=2$ ) is presented in Figure 1. Clearly, large ringing effects are present on the background, while the object relative dielectric permittivity is seriously underestimated. Figure 2 shows the corresponding result retrieved with the Banach space approach for $p=1.3$. The dielectric reconstruction given by the variable exponent Lebesgue algorithm is reported in Figure 3. As can be seen, this result is accurate in reconstructing both the target properties and the background.

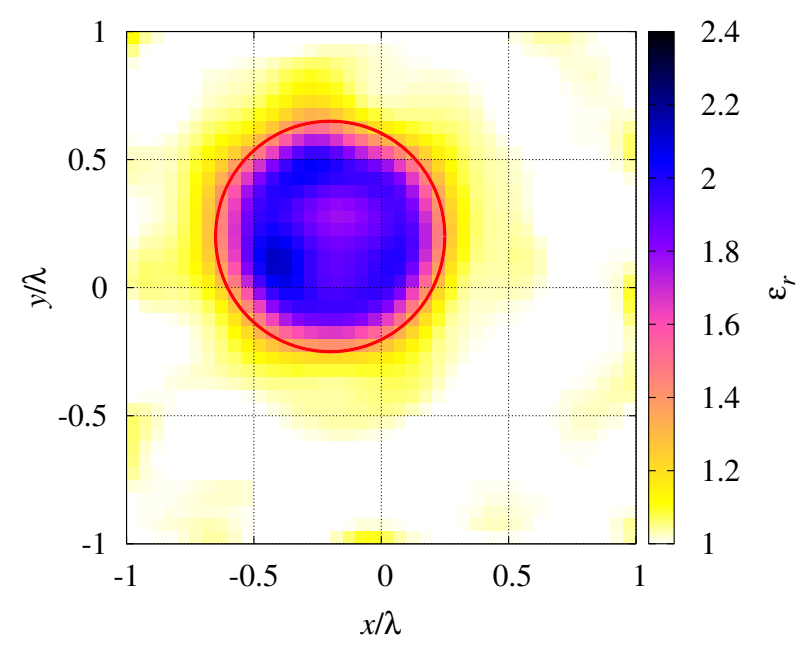

Figure 2. Reconstructed distribution of the relative dielectric permittivity $\epsilon_{r}$ inside the investigation domain. Red circle marks the true profile. Banach-space reconstruction with a constant value $p=1.3$.

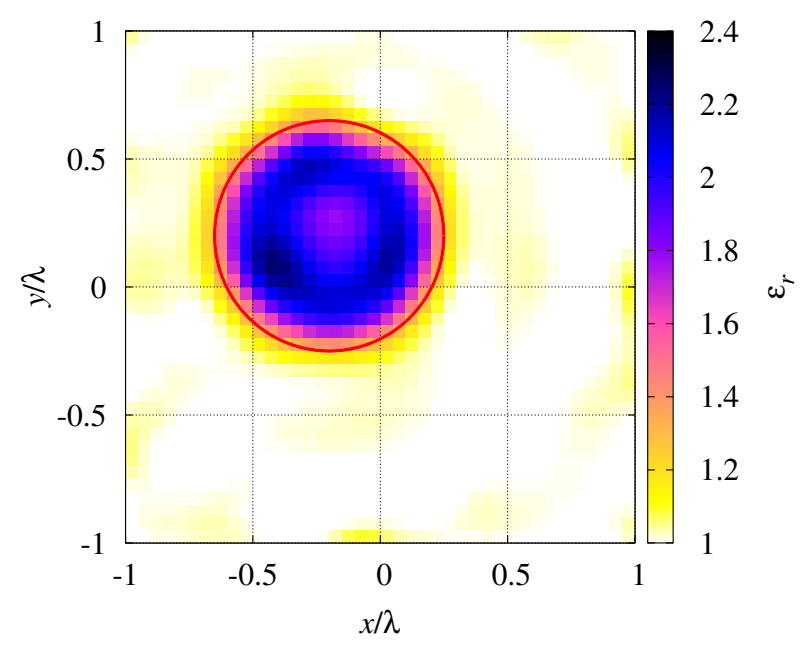

Figure 3. Reconstructed distribution of the relative dielectric permittivity $\epsilon_{r}$ inside the investigation domain. Red circle marks the true profile. Lebesgue-space reconstruction with non-constant exponents.

Furthermore, the novel approach eliminates the need of selecting the optimal value of the $p$ parameter, since the choice of a range of variation is less critical. The previous qualitative remarks are confirmed by the analysis of the average relative reconstruction errors computed in different regions of the investigation domain (whole investigation domain, cells belonging to the background and to the object regions), summarized in Table 1.

\section{Conclusions}

In this paper, an innovative microwave imaging method developed in the framework of variable exponent Lebesgue 
Table 1. Average relative reconstruction errors calculated in the whole investigation domain, in the object region and in the background region, for different reconstruction methods.

\begin{tabular}{|c|c|c|c|}
\hline \multirow{2}{*}{ Method } & \multicolumn{3}{|c|}{ Reconstruction Errors } \\
\cline { 2 - 4 } & $\begin{array}{c}\text { Whole } \\
\text { domain }\end{array}$ & $\begin{array}{c}\text { Background } \\
\text { only }\end{array}$ & $\begin{array}{c}\text { Object } \\
\text { only }\end{array}$ \\
\hline Hilbert & 0.142 & 0.127 & 0.219 \\
\hline $\begin{array}{c}\text { Banach, } \\
p=1.3\end{array}$ & 0.085 & 0.078 & 0.117 \\
\hline $\begin{array}{c}\text { Variable } \\
\text { exponent }\end{array}$ & 0.067 & 0.058 & 0.114 \\
\hline
\end{tabular}

functional spaces has been introduced. On the one hand, this method greatly improves the quality of the solution with respect to the standard Hilbert-space reconstruction algorithms. On the other hand, with respect to the more recent Banach space approaches, the particular norm that has been used here inside the minimization procedure frees the user from the problem of choosing a suitable fixed value of the norm parameter. Some preliminary results, obtained in a numerical test bench, prove the effectiveness of the proposed method.

\section{Acknowledgment}

The work of C. Estatico is partly supported by PRIN 2012 N. 2012MTE38N and GNCS-INDAM.

\section{References}

1. J. D. Shea, P. Kosmas, B. D. V. Veen, and S. C. Hagness, "Contrast-enhanced microwave imaging of breast tumors: a computational study using 3D realistic numerical phantoms," Inverse Probl., 26, 7, Jul. 2010, p. 074009.

2. K. Bialkowski, D. Ireland, and A. Abbosh, "Microwave imaging for brain stroke detection using Born iterative method," IET Microw. Antennas Propag., 7, 11, Aug. 2013, pp. 909-915.

3. G. Bozza, C. Estatico, M. Pastorino, and A. Randazzo, "Microwave imaging for nondestructive testing of dielectric structures: numerical simulations using an inexact Newton technique," Mater. Eval., 65, 9, Sep. 2007 , pp. 917-922.
4. M. Pastorino, A. Randazzo, A. Fedeli, A. Salvadè, S. Poretti, M. Maffongelli, R. Monleone, and M. Lanini, "A microwave tomographic system for wood characterization in the forest products industry," Wood Mater. Sci. Eng., 10, 1,2015 , pp. 75-85.

5. I. Catapano, A. Randazzo, E. Slob, and R. Solimene, "GPR imaging via qualitative and quantitative approaches," in Civil Engineering Applications of Ground Penetrating Radar, A. Benedetto and L. Pajewski, Eds. Springer International Publishing, 2015, pp. 239-280.

6. M. Pastorino, Microwave imaging. Hoboken, N.J.: John Wiley \& Sons, 2010.

7. A. Abubakar, T. M. Habashy, G. Pan, and M.-K. Li, "Application of the multiplicative regularized GaussNewton algorithm for three-dimensional microwave imaging," IEEE Trans. Antennas Propag., 60, 5, May 2012, pp. 2431-2441.

8. C. Estatico, M. Pastorino, and A. Randazzo, "Microwave imaging of three-dimensional targets by means of an inexact-Newton-based inversion algorithm," Int. J. Antennas Propag., 2013, Aug. 2013, p. 1-10 (407607).

9. M. Ostadrahimi, P. Mojabi, A. Zakaria, J. LoVetri, and L. Shafai, "Enhancement of Gauss-Newton inversion method for biological tissue imaging," IEEE Trans. Microw. Theory Tech., 61, 9, Sep. 2013, pp. 3424-3434.

10. C. Estatico, M. Pastorino, and A. Randazzo, "A novel microwave imaging approach based on regularization in Lp Banach spaces," IEEE Trans. Antennas Propag., 60, 7, Jul. 2012, pp. 3373-3381.

11. C. Estatico, A. Fedeli, M. Pastorino, and A. Randazzo, "Buried object detection by means of a Lp Banach-space inversion procedure," Radio Sci., 50, 1, Jan. 2015, pp. 4151.

12. C. Estatico, A. Fedeli, M. Pastorino, and A. Randazzo, "A multifrequency inexact-Newton method in Lp Banach spaces for buried objects detection," IEEE Trans. Antennas Propag., 63, 9, Sep. 2015, pp. 4198-4204.

13. P. Brianzi, F. Di Benedetto, and C. Estatico, "Improvement of Space-Invariant Image Deblurring by Preconditioned Landweber Iterations," SIAM J. Sci. Comput., 30, 3, Jan. 2008, pp. 1430-1458.

14. J. Richmond, "Scattering by a dielectric cylinder of arbitrary cross section shape," IEEE Trans. Antennas Propag., 13, 3, May 1965, pp. 334-341. 